\title{
Le Dominique Interactif
}

\author{
Valérie Ouellette \\ Université de Montréal
}

This article presents the Dominic Interactive, a structured pictorial instrument that evaluates seven mental disorders in young. The version for six to eleven years-old children is adapted to their cognitive limitations and has a lot of advantages. Despite some limitations, this instrument gives reliable information on mental health of the child. The validity and reliability of the Dominic Interactive are better than those of other traditional instruments. A complete evaluation of the child's mental health is possible if we combine the results of this instrument with information from parents and teachers. Finally, its simple and fast use makes the professional's evaluation easier. It also promotes program's evaluation and early intervention.

Cet article présente le Dominique Interactif, une bande dessinée interactive et multimédia unique, servant à évaluer sept troubles de la santé mentale chez les jeunes. La version pour les six à onze ans est adaptée aux limites cognitives des enfants et comporte plusieurs avantages. Malgré quelques limites, cet instrument permet d'obtenir des informations fiables de la part de l'enfant sur sa santé mentale. La fidélité et la validité de cet outil sont meilleures que celles des autres instruments traditionnels servant à mesurer la santé mentale des enfants. En combinant les résultats de ce questionnaire avec les informations des parents, professionnels et enseignants, le Dominique Interactif permet aussi d'effectuer une évaluation complète de la santé mentale de l'enfant. Enfin, son utilisation simple et rapide facilite l'évaluation clinique des enfants par les professionnels, permet l'évaluation de programmes d'intervention et favorise l'intervention précoce (Valla \& al., 2000).

Les problèmes de santé mentale engendrent de la souffrance et des coûts élevés dans notre société. La définition de ce qui est normal ou pathologique ne peut donc pas être laissée au jugement subjectif des individus, même s'il n'existe pas de critère étalon à la classification des troubles mentaux (Valla \& Bergeron, 1994). C'est pourquoi certains chercheurs ont développé des instruments de mesure standardisés permettant à l'enfant de donner des informations sur sa santé mentale. Une équipe a mis au point un questionnaire, nommé Dominique Interactif (Valla, 2000), permettant d'évaluer la santé mentale des jeunes. Une version a été crée pour les six à onze ans et une autre pour les adolescents. La version pour les six à onze ans fait l'objet de ce travail. Une description de l'historique de l'instrument sera d'abord présentée. Elle sera suivie d'une description et des explications relatives à la passation. Ses propriétés psychométriques, les autres versions existantes ainsi que les avantages et les limites seront ensuite présentés. Finalement, il sera question de quelques comparaisons avec d'autres instruments et de l'utilité de celui-ci.

\section{Historique de l'instrument}

Un questionnaire nommé Dominique fut créé en 1981 (1984, dans Valla, Bergeron, Bérubé, Gaudet \& St-Georges, 1994). Il s'agissait d'une bande dessinée en format papier présentant Dominique, un enfant, dans diverses situations. Plus précisément, les images illustraient les comportements du personnage, représentant 26 symptômes intériorisés et extériorisés du DSM-III (American Psychiatric Association, 1980). Dans cette première version, les enfants devaient répondre par oui ou par non à savoir s'ils se comportaient, ressentaient ou étaient comme Dominique. Afin de clarifier les interprétations des dessins pouvant être faites par les enfants, des descriptions verbales des symptômes ont été ajoutées à une nouvelle version de l'instrument (Valla, Bergeron, St-Georges, Berthiaume, 2000). L'adulte faisant 
passer le questionnaire au jeune devait donc lire une question pendant que l'enfant regardait le dessin.

Les dessins ont été vérifiés et soumis à un processus de validation. Deux cahiers, respectivement de 49 et 47 pages, sont ressortis de ce processus. Une version plus brève a ensuite été créée puisque les recherches montraient que l'instrument était trop long (Valla \& al., 2000).

\section{Description et passation}

Par la suite, les avancées technologiques ont permis de créer le Dominique interactif. Il s'agit d'une bande dessinée interactive multimédia. Comme dans les versions antérieures, un jeune personnage, nommé Dominique, est placé dans diverses situations représentant des critères pour certaines psychopathologies du DSM-IV (American Psychiatric Association, 1994). Par contre, dans cette version, l'adulte présent doit d'abord établir le sexe du personnage en sélectionnant le bouton « $F$ » pour fille ou « $G$ » pour garçon (en fonction du sexe de l'enfant) dans le menu à cet effet. L'ordinateur présente ensuite à l'enfant Dominique (soit une jeune fille ou un jeune garçon), son père, sa mère et quelques images d'introduction afin de lui expliquer la tâche. Ensuite, une série de 90 illustrations de Dominique dans différents contextes, à l'école, à la maison ou avec ces amis est présentée.

Selon les théories cognitives, le traitement de l'information et la compréhension des concepts seraient meilleurs lorsqu'il y a combinaison de stimuli visuels et auditifs que lorsque les stimuli auditifs ou visuels sont présentés séparément (Frostig \& Maslow, 1979, dans Valla \& al., 2000). Dans le Dominique Interactif, lorsqu'une image apparaît à l'écran, une question, représentant la description verbale du symptôme étudié, est donc posée à l'enfant, via les hauts-parleurs. L'enfant doit ensuite répondre en cliquant avec la souris sur le bouton oui (vert) s'il réagit comme Dominique dans une telle situation. Sinon, il doit cliquer sur le bouton non (rouge) situé au bas de l'écran (voir Figure 1). Un nouveau dessin apparaît ensuite et la séquence recommence. Avant de débuter la passation, l'adulte présent doit aviser l'enfant que ses réponses ne seront montrées à personne, pas même à ses parents, sauf s'ils rapportent des comportements mettant sa vie en danger. Il doit rester près du jeune lors de l'introduction du logiciel et se retirer par la suite, sans toutefois quitter la pièce. L'adulte peut faire passer le questionnaire à plusieurs enfants à la fois. Cependant, chacun d'eux devra porter des écouteurs et avoir un ordinateur (Windows ou McIntosh) muni du logiciel et de la disquette d'enregistrement des résultats. À la fin de la passation, l'évaluateur doit toujours vérifier la section des résultats. Une liste des problèmes de santé mentale évalués par le questionnaire y est présente avec un score total pour chacun (voir Figure 2). Un rond de couleur est adjacent aux scores de chaque problème. La couleur verte signifie que le jeune ne présente probablement pas le problème. La couleur jaune signifie que le jeune ne présente peut-être pas de problème et la couleur rouge signifie qu'il y a probablement un problème. L'évaluateur doit regarder plus spécifiquement les résultats liés aux comportements suicidaires. Si de tels comportements sont notés, l'évaluateur doit en discuter avec le jeune et si la situation est sérieuse, contacter les parents de l'enfant.

\section{Explications théoriques}

Deux systèmes de classification permettent d'étudier la santé mentale des jeunes. Il s'agit des classifications dimensionnelles et catégorielles. Dans la première, les enfants sont placés sur un continuum émotionnel ou comportemental qui va du plus normal au plus pathologique. Dans la seconde, l'enfant est placé dans la catégorie normale ou pathologique. Le DSM-IV est un exemple de ce dernier type de classification. Même si ces deux types de classification sont différents, ils sont tous deux basés sur une symptomatologie très semblable. Ils établissent aussi des scores et des seuils basés sur le nombre de symptômes (Valla \& al., 2000). Le contenu du Dominique Interactif a été basé sur le DSM-IV puisqu'il s'agit de la classification dont le consensus est le plus large en Amérique du Nord actuellement (Valla \& al.). Par contre, des aménagements ont été faits afin de contrer les critiques liées à cet instrument.

D'abord, comme le décrit Piaget dans ces théories sur les opérations concrètes, les limites cognitives des enfants de six à onze ans ne leur permettent pas de tenir compte avec précision d'éléments temporels comme la durée ou la fréquence des symptômes. Ces caractéristiques sont donc absentes dans le Dominique Interactif. Elles représentent toutefois des normes de sévérité dans le DSM-IV. Pour

Figure 1: Exemple d'une question pour évaluer les troubles de conduite au Dominique Interactif

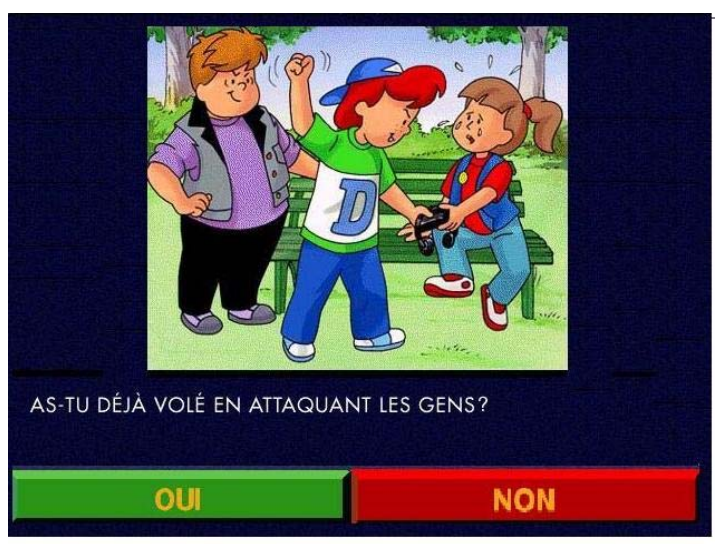


Figure 2: Exemple de la présentation des résultats au Dominique Interactif

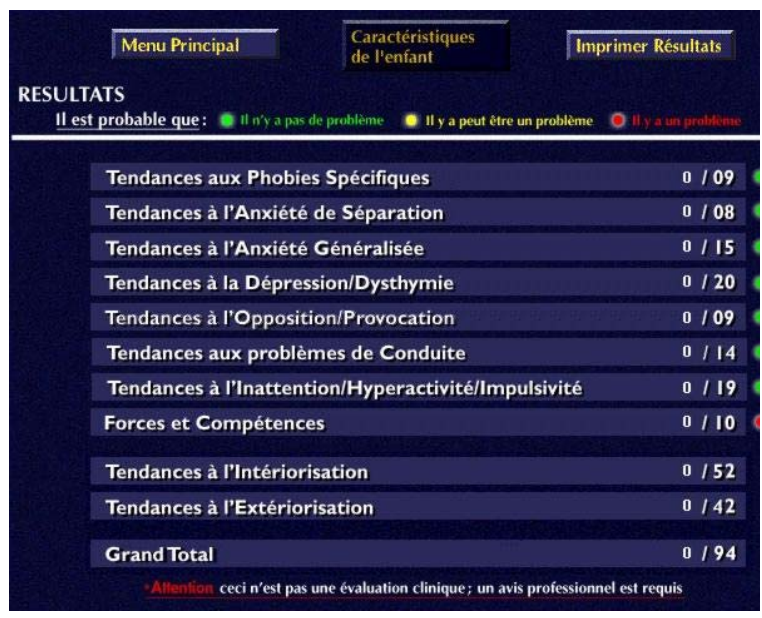

remédier à cette absence, le nombre de symptômes minimum requis afin d'identifier un trouble a été augmenté dans le Dominique Interactif. L'absence de ces critères dans l'évaluation restreint la correspondance de l'instrument avec le DSM-IV. Cependant, cela permet d'avoir une source d'information fiable.

Le Dominique Interactif n'est pas un instrument permettant de faire des diagnostics. Il propose une approximation des diagnostics selon le DSM-IV dont la prévalence est la plus élevée pour les enfants de six à onze ans. La plupart des instruments d'évaluation de la santé mentale identifient moins bien les enfants situés près du seuil de psychopathologie que ceux aux extrémités du continuum. Afin de diminuer le nombre de faux positifs et de faux négatifs, une troisième catégorie a été ajoutée. Ce questionnaire identifie donc si l'enfant évalué ne présente probablement pas, pourrait présenter ou présente probablement un problème de santé mentale (Valla \& al., 2000). Les résultats seront donc décrits comme des «scores de tendance ». En tout, sept problèmes de santé mentale sont évalués. Il s'agit de : l'anxiété généralisée, les phobies spécifiques, l'anxiété de séparation, la dépression, le déficit d'attention et l'hyperactivité, les troubles de conduite et le trouble oppositionnel. Certains symptômes font partie de l'évaluation de plus d'un problème de santé mentale. Par exemple, l'irritabilité compte dans le score de tendance de la dépression et $\mathrm{du}$ trouble oppositionnel. De plus, certains critères, comme la reconnaissance du caractère excessif de la peur ou forcer un individu à une activité sexuelle, sont trop abstraits ou inadéquats pour des enfants de cet âge et n'ont donc pas été représentés. L'instrument évalue tout de même 62 des 66 symptômes reliés à ces sept problèmes de santé mentale présents dans le DSM-IV.

Enfin, les questions de l'instrument ont été réparties afin que les images qui se succèdent n'appartiennent pas à un même trouble. La deuxième moitié du questionnaire contient les questions reliées aux problèmes les plus graves, comme la dépression et les troubles de conduite. Dix images représentant des situations positives ont été ajoutées afin de diminuer l'identification à un héros déviant et d'éviter ou d'aider à repérer les réponses systématiques par oui ou par non.

\section{Propriétés psychométriques}

Une étude complète des propriétés psychométriques du Dominique Interactif est présentement en cours auprès de 600 enfants francophones et anglophones de la région de Montréal. Des études préliminaires portant sur la fidélité test-retest ont été faites puisqu'il s'agit de la propriété psychométrique la plus difficile à obtenir chez les enfants (Valla \& al, 2000). La valeur médiane des kappas entre le test et le retest, par symptômes, va de 0,45 à 0,50. Le kappa est une statistique évaluant 1 'accord entre deux mesures. Elle examine la fiabilité des évaluations. Selon le problème évalué, les corrélations intraclasses entre le test et le retest se situent entre 0,59 (anxiété généralisée) et 0,80 (hyperactivité). Enfin, les réponses des filles ainsi que celles des enfants plus âgés sont légèrement plus stables que celles des garçons et des enfants plus jeunes.

Les propriétés psychométriques des instruments sont généralement équivalentes ou un peu meilleures en format interactif qu'en format papier (Richman, Kiesler, Weisband \& Drasgow, 1999). Les résultats des études préliminaires montrent qu'effectivement, il y a peu de différence entre le Dominique Interactif et le Dominique- $R$ (Valla \& al., 2000). Ce dernier a été évalué auprès de 340 enfants de quatre écoles de la région de Montréal.

Pour la fidélité test-retest, des analyses pour chaque échelle ainsi que pour chaque symptôme ont été effectuées. Les valeurs des kappas étaient supérieures ou égales à 0,60 pour 21 symptômes, entre 0,50 et 0,59 pour 50 autres et entre 0,40 et 0,49 pour 14 autres symptômes. Enfin, pour sept symptômes, il a été impossible de faire ces analyses puisque le nombre de réponses positives était insuffisant.

Les corrélations intraclasses entre le test et le retest pour les échelles de symptômes se situaient entre 0,71 et 0,81 selon le trouble évalué.

La validité de critère a aussi été étudiée pour le Dominique- $R$. Les cliniciens demandaient aux jeunes d'expliquer la signification de l'image, puis ils vérifiaient la correspondance avec les critères du DSM-III-R censés leur correspondre. Les valeurs des kappas des accords cliniciens/enfants vont de 0,64 (problèmes de conduite) à 0,88 (dépression) selon le trouble. Pour ce qui est des accords interjuges, les valeurs des kappas se situent entre 0,79 et 0,95 . 
Selon Valla et al. (2000), la fidélité et la validité du Dominique sont plus élevées que celles des instruments plus traditionnels d'évaluation de la santé mentale chez les enfants de six à onze ans.

\section{Seuil du Dominique Interactif}

Des seuils ont été déterminés afin d'identifier à quelle catégorie (ne présente probablement pas, pourrait présenter ou présente probablement un problème de santé mentale) l'enfant appartient au moment où il est testé (voir tableau 1). Ces seuils ont été établis en fonction du DSM-IV, et de considérations cliniques et statistiques (Valla \& al.). D'abord, les seuils de cet instrument sont proportionnels à ceux du DSM-IV. Les résultats au Dominique Interactif de 44 enfants provenant de milieux cliniques ont été comparés aux évaluations cliniques comprenant l'évaluation de l'enfant, une rencontre avec les parents et des informations du milieu scolaire. Enfin, dans une étude sur un échantillon de convenance, les participants ayant des résultats plus d'un écart type sous la moyenne ont été considérés comme déviants. Ceux ayant des résultats plus de deux écarts types sous la moyenne ont été considérés comme très déviants. Toutes ces démarches ont mené aux seuils du Dominique Interactif. Approximativement $20 \%$ des enfants de la population ont été identifiés par le seuil inférieur et pourraient donc présenter un problème. Entre 5\% et $10 \%$ des enfants de la population présenteraient probablement un problème selon le seuil supérieur établi. La prévalence observée dans les études des populations identifie un peu moins de jeunes. Les enfants obtenant des résultats positifs avec le Dominique Interactif ne présentent donc pas nécessairement une psychopathologie suffisamment grave pour avoir besoin d'une prise en charge (Valla \& al., 2000). Il est recommandé aux professionnels utilisant cet instrument de tenir compte du but de l'évaluation, du contexte de l'enfant et de toutes autres données pertinentes à sa disposition (Valla \& al.).

\section{Autres versions du Dominique Interactif}

Selon Rosenberg et Simmons (1972), les enfants noirs utilisent des groupes d'individus noirs plutôt que blancs comme référence lorsqu'ils se comparent. De plus, à partir de l'âge de trois ou quatre ans, les enfants sont conscients des différences ethniques et culturelles (Porter, 1971). Comme le Dominique utilise des stimuli visuels, les auteurs ont tenu compte de la diversité ethnique. Des versions représentant des individus hispaniques, afro-américains et asiatiques ont donc été créées. Le Dominique interactif est très récent. Les versions interactives pour hispaniques ou afro-américains sont présentement en préparation. Cet instrument est toutefois disponible en français européen, canadien français, espagnol européen, espagnol mexicain, anglais et en allemand.

Aux États-Unis, un taux plus élevé de troubles émotionnels et de comportements a été rapporté chez les minorités ethniques. Une étude a donc été menée auprès $\mathrm{d}$ 'afro-américains afin de vérifier la fidélité de la version du Dominique-R, appelée «Terry» (Bidaut-Russel, Valla, Thomas, Bergeron \& Lawson, 1998). Les résultats font ressortir une bonne fidélité en ce qui concerne le diagnostic. La fidélité test-retest est aussi très élevée avec des valeurs entre $88,8 \%$ et $97,2 \%$ dépendamment du trouble évalué. La fidélité de l'échelle de symptômes était aussi très bonne pour les sept troubles évalués. Enfin, la consistance interne était de bonne à excellente pour six des sept diagnostics mais modérée pour l'anxiété généralisée. Donc bien qu'il reste à vérifier la validité de cette version de l'instrument, celui-ci semble prometteur.

Une autre étude, plus récente, a été faite afin de vérifier si la version interactive du Dominique était appropriée pour les enfants français malgré les différences culturelles existantes entre les cultures française et nord américaine. Le Dominique Interactif a été passé à 403 enfants français, dont 150 patients de cliniques externes ayant reçu un diagnostic clinique. Une différence est ressortie pour chacune des échelles de symptômes et pour chaque estimé de prévalence. Cet instrument discrimine donc bien entre le groupe d'enfants référés et non référés De plus, l'instrument discrimine bien entre les enfants référés ayant ou non reçu un diagnostic clinique à l'exception $\mathrm{du}$ trouble oppositionnel. Par contre, quelques mots devraient être changés afin que les enfants comprennent mieux les questions. Malgré tout, ces données sont encourageantes et montrent que l'instrument peut être approprié à plusieurs cultures.

\section{Avantages}

Plusieurs avantages sont reliés au Dominique Interactif (Valla, Bergeron, Smolla, 2000). D'abord, le format interactif de l'instrument est assez simple pour que l'enfant puisse l'utiliser et suffisamment attrayant pour maintenir l'attention du jeune. Cela induit aussi moins de désirabilité sociale qu'une entrevue entre l'adulte et le jeune. Ensuite, la durée de passation moyenne pour un jeune de six à onze ans est de 10-15 minutes, ce qui est assez bref pour maintenir l'attention du jeune. L'intérêt de l'enfant est aussi stimulé puisqu'il répond lui même aux questions. La passation de l'instrument par l'enfant lui-même diminue aussi le risque de biais de l'évaluateur et l'influence de l'environnement. Cela rend aussi les résultats des jeunes plus comparables. Les images font que les résultats ne sont pas basés seulement sur le vocabulaire de l'enfant. Enfin, les réponses de l'enfant sont automatiquement enregistrées sur une disquette, ce qui permet d'obtenir les résultats 
immédiatement à la suite de la passation.

\section{Limites}

Des recherches montrent que les jeunes identifient bien leurs problèmes intériorisés comme la dépression, mais que les troubles extériorisés seraient mieux identifiés par les parents ou les professeurs (Edelbrock, Costello, Dulcan, Calabro-Conover \& Kala, 1986). Comme les informations proviennent entièrement du point de vue du jeune dans cet instrument, il ne permet pas une évaluation complète de sa santé mentale. Par exemple, les problèmes de conduite et d'opposition risquent d'être auto-évalués à la baisse par les jeunes. En effet, par désirabilité sociale, plusieurs enfants ont appris à mentir pour cacher leurs comportements antisociaux (Valla \& al., 2000). Afin de compléter l'évaluation, on doit donc combiner les informations recueillies avec celles provenant des parents ou professeurs (Jensen \& al., 1999).

Le Dominique Interactif partage aussi quelques limites des instruments standardisés puisqu'il en est un. D'abord, les troubles mentaux sont des phénomènes extrêmement complexes et l'instrument n'offre qu'une description sommaire et sans caractère idiosyncrasique. De plus, la symptomatologie n'est pas suffisante si une décision doit être prise (Trickett, 1996). Une évaluation de l'environnement, de l'histoire personnelle et du contexte dans lequel vit le jeune est alors nécessaire. L'interprétation des résultats ne peut être effectuée que par une personne ayant les connaissances théoriques des psychopathologies évaluées ainsi que des instruments standardisés.

Puisqu'il s'agit du premier instrument alliant des stimuli temporels et des stimuli auditifs, il existe peu d'information sur la validité prédictive. De plus, il est difficile de comparer cet instrument aux autres évaluant la santé mentale.

Enfin, la section résultats du logiciel permet d'obtenir les résultats totaux du questionnaire. Par contre, il n'existe pas de lien entre le Dominique Interactif et un logiciel comme excel. Afin d'effectuer des analyses statistiques, il est donc nécessaire d'imprimer les résultats et de les entrées manuellement dans une base de données. Des mesures doivent alors être prises afin d'éviter les erreurs dans la saisie des données.

\section{Comparaisons avec d'autres instruments}

Très peu d'instruments évaluent la santé mentale chez les jeunes de six à onze ans. De plus, la plupart de ces instruments sont des entrevues semi-structurées basées sur le jugement clinique de l'interviewer. Les résultats de ces entrevues ne sont pas assez précis et ne permettent pas de confirmer leur validité et leur fidélité. Par exemple, les valeurs test-retest de l'une de ces entrevues, nommée KiddieSADS (Orvaschel \& Puig-Antich, 1987) varient entre 0,24 et
0,70 (Chambers \& al., 1985). Les entrevues structurées, comme le Diagnostic Interview Schedule for Children (Costello \& Edelbrock, 1985), présentent aussi des problèmes puisque les corrélations test-retest pour ces instruments sont faibles (Edelbrock, Costello, Dulcan, Kalas \& Conover, 1985). Les limites cognitives des enfants de cet âge peuvent expliquer en partie la difficulté des instruments à évaluer adéquatement la santé mentale des jeunes de six à onze ans. En effet, l'attention et la compréhension des jeunes de cet âge sont limitées. Cela peut leur donner de la difficulté à terminer une longue tâche ou à comprendre le vocabulaire dans les questions qui leur sont posées. Un manque d'intérêt envers la tâche peut aussi en résulter (Valla, Bergeron, Bérubé, Gaudet \& St-Georges, 1994).

Toutefois, quatre autres instruments standardisés ont été construits dans le but d'évaluer la santé mentale des jeunes avec l'aide d'images. L'un deux, nommé Preschool Symptom Self-Report (Martini, Strayhorn \& Puig-Antich, 1990) évalue les symptômes dépressifs des enfants de trois à cinq ans. Le Levonn (Richters, Martinez \& Valla, 1990) étudie la détresse des enfants victimes de stress posttraumatique. Le Pictorial Instrument for Children and Adolescents évalue la symptomatologie des jeunes hospitalisés (Ernst, Godfrey, Silva, Pouget \& Welkowitz, 1994). Enfin, le Darryl porte sur le stress post-traumatique (Neugebauer, Wasserman, Fisher, Kline, Geller \& Miller, 1999). Les comparaisons du Dominique Interactif avec ces instruments sont limitées puisque la symptomatologie ou les groupes d'âge étudiés ne sont pas équivalents (Valla \& al., 2000). Ces instruments sont aussi plus spécifiques à une problématique que ne l'est le Dominique Interactif. Celui-ci offre donc une évaluation plus complète de la santé mentale des jeunes tout en tenant compte des limites cognitives des enfants.

\section{Utilité}

En milieu clinique, cet instrument offre l'opportunité aux cliniciens de faire une revue de la santé mentale des jeunes de façon assez simple, intéressante pour l'enfant et rapide. Les enfants de six à onze ans sont souvent référés pour des troubles de comportements. Le Dominique Interactif aide alors à détecter des psychopathologies pouvant être moins évidentes ou masquées par ces troubles (Valla \& al., 2000). Par contre, l'instrument ne remplace pas une évaluation clinique. Il sert plutôt d'appui à celle-ci.

Cet instrument peut aussi servir en contexte de recherche, de prévention et d'intervention. Par exemple, afin de prévenir l'apparition et l'aggravation de psychopathologies et des difficultés scolaires associées chez les enfants du primaire, le conseil scolaire de l'île de Montréal a subventionné une étude (Bergeron \& Valla, 2000). Les objectifs étaient de dépister les troubles de santé 
mentale et les indicateurs de risque ainsi que d'intégrer ce dépistage avec l'intervention et la prévention. Dans quatre milieux défavorisés de Montréal, 120 enfants de quatre à huit ans ont été évalués. Pour les 79 enfants de six à huit ans, le Dominique Interactif a été utilisé pour évaluer leur santé mentale. Afin de décider du type d'intervention à effectuer, un Groupe Multidisciplinaire Intersectoriel (GMI), composé de psychiatres, de travailleurs sociaux et de représentants du milieu scolaire, ont discuté de certains cas. Les résultats montrent que 59,5\% des enfants évalués à l'aide du Dominique Interactif présentaient au moins un trouble. L'anxiété de séparation, l'anxiété généralisée et les troubles de conduite et d'opposition étaient les plus fréquents. On retrouvait aussi une comorbidité chez 33\% des enfants. La plupart de ceux-ci ont été orientés vers la pédopsychiatrie suite aux discussions du GMI. Selon les conclusions de cette étude, le dépistage des problèmes de santé mentale chez les enfants de milieux défavorisés est faisable. L'étude montre aussi que le pourcentage d'enfants de six à huit ans présentant au moins un trouble de la santé mentale est quatre fois plus élevé que dans la population des enfants du Québec. Les auteurs suggèrent que la concentration des ressources dans ce milieu devrait être proportionnelle à la quantité de troubles qu'on y retrouve, comparativement avec les autres milieux. Les auteurs ont aussi fait quatre recommandations pour orienter les études futures en prévention des problèmes de santé mentale en milieu scolaire. L'une d'elles est l'utilisation d'instruments standardisés informatisés pour le dépistage à grande échelle parce que cela uniformise la définition des troubles de santé mentale et facilite le recueil des données, réduit le nombre d'erreurs de codification et produit immédiatement un rapport des résultats du dépistage. On peut déduire de cette recommandation que les chercheurs ont su apprécier les qualités offertes par le Dominique Interactif et que l'instrument a été efficace dans ce contexte.

Le Dominique Interactif permet aussi d'évaluer des programmes $d$ 'intervention visant les élèves dès le primaire puisqu'il permet d'obtenir des informations stables sur la santé mentale des enfants à partir de l'âge de six ans. Cet instrument est donc utile en recherche évaluative ainsi qu'en recherche épidémiologique.

Finalement, le Dominique Interactif est un instrument unique d'évaluation de sept troubles de la santé mentale des jeunes. La version pour les six à onze ans est adaptée aux limites cognitives des enfants et comporte plusieurs avantages. Il est impossible de nier certaines de ses limites dont la plupart sont communes à tous les instruments standardisés. Malgré tout, cet instrument permet d'obtenir des informations fiables de la part de l'enfant sur sa santé mentale. En combinant les résultats avec les informations des parents, professionnels et enseignants, le Dominique
Interactif permet aussi d'effectuer une évaluation complète de la santé mentale de l'enfant. Enfin, son utilisation simple et rapide facilite l'évaluation clinique des professionnels, permet l'évaluation de programmes d'intervention et favorise l'intervention précoce (Valla \& al., 2000).

\section{Références}

American Psychiatric Association. (1980). Diagnostic and Statistical Manual of Mental Disorders, Third Edition (DSMIII). Washington, DC : American Psychiatric Press.

American Psychiatric Association. (1987). Diagnostic and Statistical Manual of Mental Disorders, Third Edition Revised (DSM-III-R). Washington, DC: American Psychiatric Press.

American Psychiatric Association. (1994). Diagnostic and Statistical Manual of Mental Disorders, Fourth Edition (DSM-IV). Washington, DC : American Psychiatric Press.

Bergeron, L. \& Valla, J.P. (2000). Dépistage des problèmes de santé mentale et des indicateurs de risque dans les écoles primaires de milieux défavorisés de l'île de Montréal. Unisson, 10, 12.

Bidaut-Russel, M., Valla, J.P., Thomas, J.M., Bergeron, L. \& Lawson, E. (1998). Reliability of the Terry: A mental health cartoon-like screener for african-american children. Child Psychiatry and Human Development, 28 (4), 250-263.

Chambers, W.J., Puig-Antich, J., Hirsch, M., Paez, P., Ambrosini, P.J., Tabrizi, A. \& Davies, M. (1985). The assessment of affective disorders in children and adolescents by semistructures interview. Archives of General Psychiatry, 42, 696-702.

Costello, E.J. \& Edelbrock, C.S. (1985). Detection of psychiatric disorders in pediatric primary care: A preliminary report. Child Psychiatry, 24, 771-774.

Edelbrock, C., Costello, A.J., Dulcan, M.K., Kalas R. \& Conover, N.C. (1985).Age differences in the reliability of the psychiatric interview of the child. Child Development, 56, 265-275.

Edelbrock, C., Costello, A., Dulcan, M.K., Calabro-Conover, N. \& Kala, R. (1986). Parent-child agreement on child psychiatric symptoms assessed via structured interview. Journal of Child Psychology and Psychiatry, 27, 181-190.

Ernst, M., Godfrey, K.A., Silva, R.R., Pouget, E.R. \& Welkowitz, J. (1994). A new pictorial instrument for child and adolescent psychiatry: a pilot study. Psychiatry Research, 51, 87-104.

Frostig, M. \& Maslow, P. (1979). Neuropsychological contributions to education. Journal of Learning Disabilities, 12, 40-54.

Jensen, P., Rubio-Stipec, M., Canino, G., Bird, H., Dulcan, M., Schwab-Stone, M. \& Lahey, B. (1999). Parent and child contribution to diagnosis of mental disorder: are 
both informants always necessary? Journal of the American Academy of Child and Adolescent Psychiatry, 32, 397-406.

Martini, D.R., Strayhorn, J.M. \& Puig-Antich, J. (1990). A symptom self-report measure for preschool children. Journal of the American Academy of Child and Adolescent Psychiatry, 29, 594-600.

Neugebauer, R., Wasserman, G.A., Fisher, P.W., Kline, J., Geller, P.A. \& Miller, L.S. (1999). Darryl, a cartoon-based measure of cardinal posttraumatic stress symptoms in school-age children. American Journal of Public Health, 89, 758-761.

Orvaschel, H. \& Puig-Antich, J. (1987). Schedule for affective disorder and schizophrenia for scool age children. Pittsburgh : Western Psychiatric Institute and Clinic.

Porter, J.D.W. (1971). Black child, white child: the development of racial attitudes. Cambridge, MA : Harvard University Press.

Richman, W.L., Kiesler, S., Weisband, S. \& Drasgow, F. (1999). A meta-analytic study of social desirability distortion in computer-administered questionnaires, traditional questionnaires, and interviews. Journal of Applied Psychology, 84, 754-775.

Richters, J.E., Martinez, P. \& Valla, J.P. (1990). Levonn: A cartoon-based structured interview for assessing young children's distress symptoms. National Institute of Mental Health.

Rosenberg, M. \& Simmons, R.G. (1972). Black and white self- esteem : the urban school child. Washington, DC : American Sociological Association.

Trickett, E.J. (1996). A future for community psychology: The contexts of diversity and the diversity of contexts. American Journal of Community Psychology, 24, 209-234.

Valla, J.P. (2000). Instruction manual for the Dominic Interactive. In : Dominic Interactive, CD-ROM, DIMAT, Montreal.

Valla, J.P., Bergeron, L. (1994). L'épidémiologie de la santé mentale de l'enfant et de l'adolescent. Paris, PUF, $128 \mathrm{p}$.

Valla, J.P., Bergeron, L., Bérubé, H., Gaudet, N. \& StGeorges, M. (1994). A structures pictorial questionnaire to assess DSM-III-R-Based diagnoses in children (6-11 years) : Development, validity, and reliability. Journal of Abnormal Child Psychology, 22 (4), 403-423.

Valla, J.P., Bergeron, L. \& Smolla, N. (2000). The Dominic-R : A pictorial interview for 6 to 11 years old children. Journal of the American Academy of Child and Adolescent Psychiatry, 39 (1), 85-93.

Valla, J.P., Bergeron, L., St-Georges, M. \& Berthiaume, C. (2000). Le Dominic interactif: présentation, cadre conceptuel, propriétés psychométriques, limites et utilisations. Revue Canadienne de Psycho-Éducation, 29 (2), 327-347.

Received April 15, 2005. Accepted September 15, 2005

Tableau 1. Seuil du Dominique Interactif et du DSM-IV

\begin{tabular}{|c|c|c|c|c|c|c|}
\hline \multirow[b]{3}{*}{$\begin{array}{c}\text { Problème de santé } \\
\text { mentale (Tendance) }\end{array}$} & \multicolumn{4}{|c|}{ Dominique Interactif } & \multicolumn{2}{|c|}{ DSM-IV } \\
\hline & \multirow[b]{2}{*}{$\begin{array}{l}\text { Nombre de } \\
\text { symptômes } \\
\text { décrits }\end{array}$} & \multicolumn{3}{|c|}{ Nombre de symptômes } & \multirow[b]{2}{*}{$\begin{array}{c}\text { Nombre de } \\
\text { symptômes } \\
\text { décrits }\end{array}$} & \multirow{2}{*}{$\begin{array}{l}\text { Nombre de } \\
\text { symptômes } \\
\text { requis pour } \\
\text { un diagnostic }\end{array}$} \\
\hline & & $\begin{array}{c}\text { Probablement } \\
\text { pas de } \\
\text { problème }\end{array}$ & $\begin{array}{c}\text { Peut-être } \\
\text { un } \\
\text { problème }\end{array}$ & $\begin{array}{l}\text { Probablement } \\
\text { un problème }\end{array}$ & & \\
\hline Opposition & 9 & $0-4$ & $5-6$ & $7-9$ & 8 & 4 \\
\hline $\begin{array}{l}\text { Problèmes de conduite } \\
\text { Déficit de l'attention/ }\end{array}$ & 14 & $0-2$ & $3-5$ & $6-14$ & 15 & 3 \\
\hline hyperactivité & 19 & $0-10$ & $11-13$ & $14-19$ & 9 & 6 \\
\hline Angoisse de séparation & 8 & $0-4$ & 5 & $6-8$ & 8 & 3 \\
\hline Anxiété généralisée & 15 & $0-9$ & $10-11$ & $12-15$ & & \\
\hline Phobies spécifiques & 9 & $0-2$ & $3-4$ & $5-9$ & \multicolumn{2}{|c|}{5 critères de gravité } \\
\hline Dépression & 20 & $0-10$ & $11-13$ & $14-20$ & 9 & 5 \\
\hline Forces et competences & 10 & $8-10$ & 7 & $0-6$ & & \\
\hline
\end{tabular}

*Informations tirées de Valla, Bergeron, St-Georges et Berthiaume, 2000. 